\title{
Prognose de falhas em um reator tubular com escoamento pistonado utilizando
} sistemas imunológicos artificiais

\section{Failure prognosis in a piston flow reactor of the tubular reactor using artificial immune systems}

Article Info:

Article history: Received 2021-04-15 / Accepted 2021-05-04/ Available online 2021-05-04

doi: 10.18540/jcecvl7iss2pp12366-01-10e

Fábio Roberto Chavarette

ORCID: https://orcid.org/0000-0001-5376-3392

UNESP - Univ. Estadual Paulista - Departamento de Engenharia, Física e Matemática do Instituto de Química, Brasil

E-mail: fabio.chavarette@unesp.br

Roberto Outa

ORCID: https://orcid.org/0000-0002-8649-1722

Faculdade de Tecnologia de Araçatuba - Faculdade de Tecnologia de Lins, Brasil

E-mail: roberto.outa@fatec.sp.gov.br

Aparecido Carlos Gonçalves

ORCID: https://orcid.org/0000-0001-5376-3392

UNESP - Univ. Estadual Paulista - Faculdade de Engenharia de Ilha Solteira, Departamento de

Engenharia Mecânica, Brasil

E-mail: aparecido.carlos@unesp.br

\section{Resumo}

A indústria 4.0 utiliza princípios de novas tecnologias para obter resultados de melhor qualidade e produtividade, o qual a computação inteligente é uma delas. Neste trabalho é proposto uma metodologia inteligente em que o sistema imunológico artificial separa o sinal em grupamentos e determina a classificação baseado em prognose de falhas pelo grau de severidade de um reator tubular com escoamento pistonado. O processo é desenvolvido da seguinte forma: basicamente, após a obtenção dos sinais de vibração do reator através de um modelo numérico, é utilizada a transformada rápida de Fourier para transformar os sinais no domínio da frequência. Posteriormente, um sistema imunológico artificial de seleção negativa realiza o diagnóstico, identificando e classificando as falhas. A motivação da aplicação desta metodologia é o processo de fiscalização de estruturas, a fim de identificar e caracterizar as falhas, bem como tomar decisões visando evitar acidentes ou desastres. Os resultados demonstram a robustez e precisão da metodologia para a aplicação proposta.

Palavras-chave: Sistemas Imunológicos Artificiais. Reator. Monitoramento.

\section{Abstract}

Industry 4.0 uses principles of new technologies to achieve better quality and productivity results, which intelligent computing is one of them. In this work is proposed an intelligent methodology in which the artificial immune system sequesters the signal into groups and determines the classification based on failure prognose by the degree of severity of a tubular reactor with piston flow. The process is developed as follows: basically, after obtaining the vibration signals from the reactor through a numerical model, the Fourier rapid transform is used to transform the signals in the frequency domain. Later, a negatively select artificial immune system performs the diagnosis, identifying and classifying failures. The motivation for the application of this methodology is the process of supervision of structures, in order to identify and characterize failures, as well as make 
decisions aimed at avoiding accidents or disasters. The results demonstrate the accuracy and the robustness of the methodology for the reactor operation.

Keywords: Artificial Immune Systems. Reactor. Reliability. SHM. Monitoring.

\section{Introdução}

Com o passar do tempo à tecnologia tem evoluído drasticamente proporcionando às empresas em geral uma maior eficiência. A quarta revolução industrial deu inicio no ano de 2011 por meio de investimentos em novas tecnologias e nas frequentes inovações sendo denominada de Indústria 4.0 sendo o termo relacionado à evolução das fábricas para fábricas inteligentes que integram sistemas virtuais e físicos trazendo grandes benefícios como a criação de novos modelos de trabalho, controle em tempo real da produção, otimização de recursos naturais e redução de erros (Popkova et al., 2019; Kelly, 2019).

Diante desta revolução, nas plantas industriais têm ocorrido mudanças tanto em processos produtivos como na disponibilidade de novos produtos através da automatização e da utilização de robôs. Essa nova realidade dá origem aos sistemas ciber-físicos, mesclando sistemas computacionais e sistemas físicos para as operações de monitoramento, coordenação com o objetivo de transformar a interação entre o ser humano com o mundo físico. Essa abordagem permite um sensoriamento dos componentes como objetivo principal de entender o estado da vida útil e criterizar sua condição sobre um aspecto de danos e falhas (Chen \& Li, 2018; Kelly, 2019).

As empresas que adotam um sistema de sensoriamento têm a vantagem de gerenciar informações estipulando as condições de redução de custos na linha de produção, formando um conceito de agilidade na produção, efetividade de mão de obra especializada, e até mesmo, a aplicação de técnicas gerenciais para a melhora da qualidade junto à nova indústria 4.0 (Chen \& $\mathrm{Li}, 2018$ ). Os sistemas de monitoramento e diagnósticos aproximam-se dos sistemas ciber-físicos devido ao desenvolvimento de novas tecnologias e dos métodos de processamento e interpretação com o objetivo de analisar a integridade de estruturas de modo eficiente impedindo que catástrofes e/ou acidentes aconteçam, certificando que vida não se perdão e impedindo prejuízos econômicos (Popkova et al., 2019; Chen \& Li, 2018).

Utilizando das informações fornecidas pelo processo de automação (sensores distribuídos), é possível realizar o controle e monitoramento da planta industrial com inteligência artificial, tornando a máquina habilitada a tomar decisões para reduzir custos, potencializar o lucro e preservar a vida (Franco et al., 2009). Podemos citar trabalhos que utilizam o princípio de monitoramento por sensoriamento tais como, a utilização da aplicação de técnicas de structural health monitoring (SHM) aplicado a artificial immune systems (AIS), o qual foram analisados diferentes sinais de um rotor dinâmico e determinados a prognose e detecção de falhas deste sistema dinâmico. O resultado demonstrado foi considerado de sucesso pela robustez, distribuição e classificação adequada dos sinais (Outa et al., 2020). Neste outro trabalho, a aplicação de prognose e detecção de falhas utilizando artificial immune systems (AIS) foi feita em um motor diesel em campo aberto. O resultado demonstrado, possibilitou a condição da identificação das falhas pelo método de vibração e acústico, e consequentemente, a classificação e prognose dos diferentes sinais coletados (Outa et al., 2021).

O reator químico é o centro dos processos químicos sendo essencial no qual a alimentação é convertida nos produtos desejados e é o local do processo onde se possui maior valor agregado (os alimentos de menor valor são convertidos em produtos de maior valor), seu projeto e operação determinam o sucesso ou o fracasso de todo o processo. No processo geral, as matérias-primas são entregues ao reator químico na temperatura, pressão e concentrações de espécies apropriadas (Stephanopoulos, 1983). Diante dessa informação esse trabalho tem como objetivo desenvolver um sistema de monitoramento inteligente da integridade do reator baseado em sistemas imunológicos artificiais para que o mesmo possa operar em máxima produção e condições de segurança identificando falhas no comportamento. 


\section{Sistemas Imunológicos Artificiais.}

O Sistema Imunológico Artificial, representa uma classe ampla da computação inteligente que tem por princípio a implementação de algoritmos que representam o processamento e a classificação de dados através do funcionamento do sistema imunológico humano. Assim tais algoritmos configuram ao SIA a capacidade e sucesso na implementação (Forrest et al., 1994; Lima et al., 2017; Farrar \& Worden, 2013).

Delineado pelo SIA, o algoritmo de Seleção Negativa, baseia-se no processo de seleção que ocorre no interior do Timo. Esta operação trabalha na diferenciação das células próprias e não próprias do corpo humano. Assim o ANS detecção as mudanças de estados de sistemas segmentados em duas fases de funcionamento: Censoriamento e Monitoramento (Forrest et al., 1994).

A condição especifica na fase de geração de detectores, provém da condição de que a diferentes informações podem surgir criando variedade nas informações que estão sendo analisadas. Com isso, a restrição da detecção, permite que o algoritmo determine com exatidão a autenticação da informação que se deseja. Neste caso, cada detector é considerado uma string que não corresponde a nenhum dado protegido. A figura 1 mostra a fase de censoriamento.

A fase de censoriamento tem por finalidade determinar um conjunto de detectores aleatórios a partir do conjunto de cadeias próprias. O fluxograma da figura 1 ilustra o descrito (Forrest et al., 1994; Bradley \& Tyrrell, 2002).

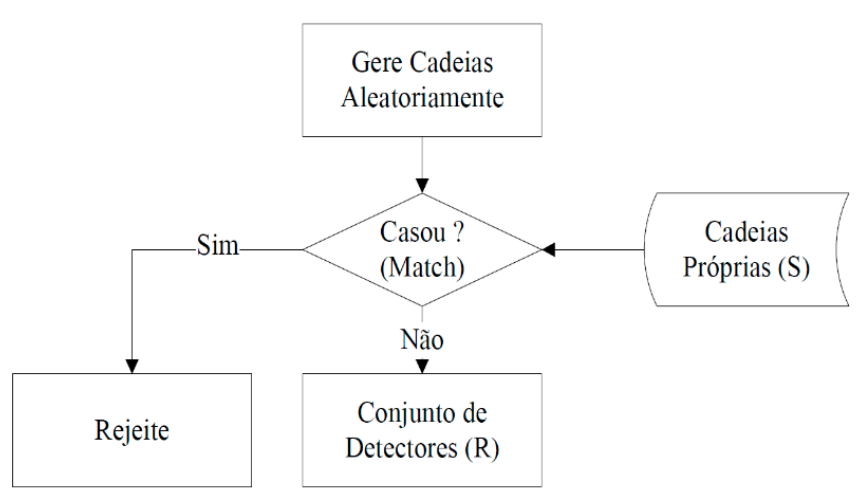

Figura. 1. Fluxograma da Fase de Censoriamento.

A fase seguinte resume-se em avaliar a integridade da estrutural a partir do conjunto de detectores formado na etapa anterior. O fluxograma desta etapa está exposto na Figura 2. Em suma, o sinal em analise é comparado com o conjunto de detectores verificando a afinidade entre os mesmos (Forrest et al., 1994; Bradley \& Tyrrell, 2002).

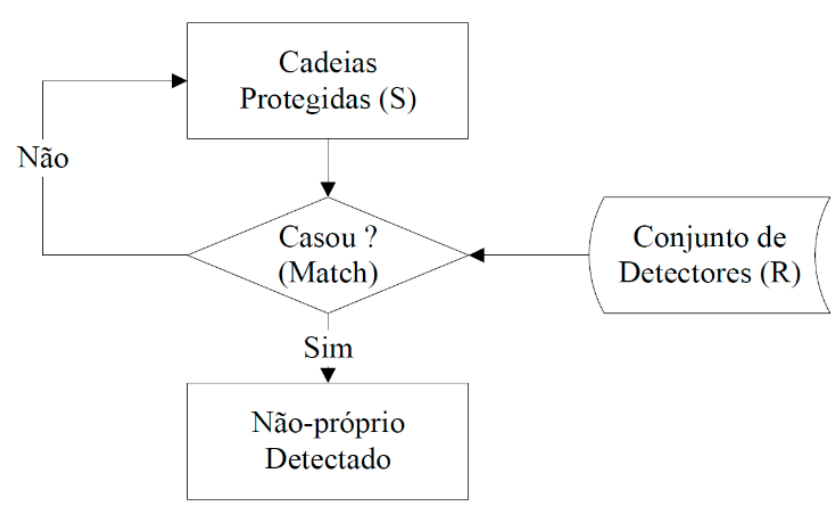


Figura. 2. Fluxograma da Fase de Monitoramento.

Para que seja feita a analise entre os sinais, um critério de casamento é utilizado, sendo que este pode ser parcial ou perfeito. Num casamento perfeito, os sinais são perfeitamente iguais, ou seja, cada posição apresenta o mesmo valor para todos os sinais em questão. Porém, no casamento parcial apenas uma porcentagem de posições deve ser maior ou igual a um valor estipulado, sendo este valor chamado de taxa de afinidade.

No caso, neste trabalho foi escolhido utilizar o conceito de casamento parcial e afinidade proposto por Bradley \& Tyrrel (2002), pois os valores utilizados são reais. Pode-se definir a taxa de afinidade como a semelhança necessária para que ocorra o casamento parcial entre dois sinais ou cadeias (Forrest et al., 1994; Bradley \& Tyrrell, 2002). Em Bradley \& Tyrrel (2002), define-se a taxa de afinidade (TAf) como:

$T A f=\left(\frac{A n}{A t}\right) * 100$

sendo TAf a taxa de afinidade; An o número de cadeias normais no problema (cadeias próprias) e At o número total de cadeias no problema (cadeias próprias e não-próprias). Além da taxa de afinidade, para analisar os padrões de cadeia é utilizado a Afinidade total, definida como:

$A f_{T}=\frac{\sum_{i=1}^{L} V c}{L} * 100$

em que $A f_{T}$ é a porcentagem de afinidade entre os padrões analisados; $L$ a quantidade total de variáveis; $V c$ as variáveis casadas e $\sum_{i=1}^{L} V c$ a somatória de sinais casados. Assim, caso $A f_{T}$ for maior o igual a TAf ocorre o casamento perfeito e as cadeias são consideradas semelhantes. Por outro lado, se a $A f_{T}$ for menor que a $T A f$, o detector não reconhece a cadeia, e logo não ocorre o casamento entre os padrões (Bradley \& Tyrrell, 2002; Lima et al., 2017; Moro et al., 2019).

\section{Modelo Numérico}

Os reatores químicos são equipamentos das mais variadas formas e tamanhos onde acontecem reações em escala industrial para transformação de matérias-primas em produtos comercializáveis. Este trabalho apresenta o modelo matemático do reator tubular com escoamento pistonado (PFR), para se obter resultados rápidos e seguros sem a realização de testes em uma planta real (Fogler, 2012; Stephanopoulos, 1983). Uma introdução ao problema que se propõe neste trabalho é ilustrada na Figura 3.

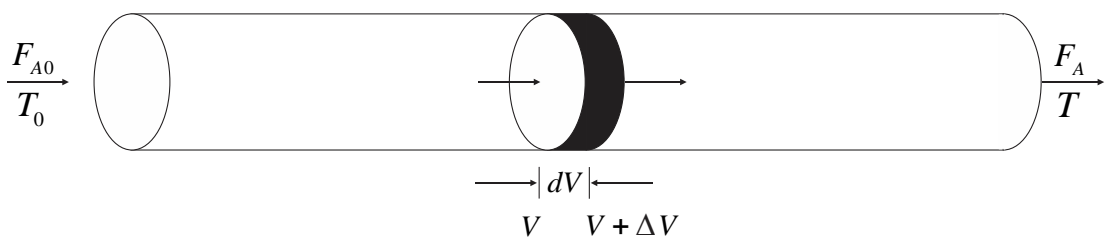

Figura 3 - Reator pistonado com escoamento uniforme (Fogler, 2012).

Modelando o problema ilustrado na Figura 1, o reator PFR, faz-se um balanço molar para o reagente A, utilizando a lei de conservação da matéria (Acúmulo - Entra - Saída + Gerado Consumido) e considerando que não há acúmulo nem geração (Fogler, 2012; Stephanopoulos, 1983). 
$F_{A}-\left(F_{A}+d F_{A}\right)-\left(-\tau_{A}\right) d V=0$

sendo

$d F_{A}=d\left[F_{A 0}\left(1-X_{A}\right)\right]=-F_{A 0} d X_{A}$

Substituindo a equação (4) em (3),

$-\tau_{A} d V=F_{A 0} d X_{A}$

Rearranjando a equação (5), têm-se a taxa de variação da conversão do reagente $\mathrm{A}$ em função do volume:

$F_{A 0} \frac{d X}{d V}=-\tau_{A}$

Como a reação ocorre em fase líquida a vazão volumétrica constante pode-se expressar a lei da velocidade de reação em função da concentração inicial de $\mathrm{A}$, da constante cinética (k) e de equilíbrio $\left(\mathrm{K}_{\mathrm{c}}\right)$, visto que a reação é reversível, fazendo:

$C_{A}=C_{A 0}\left(1-X_{A}\right)$

$\mathrm{e}$

$C_{B}=C_{A 0} X_{A}$

substituindo as equações (7) e (8) em $-\tau_{A}=k\left(C_{A}-\frac{C_{B}}{k_{c}}\right)$, tem-se

$-\tau_{A}=k C_{A 0}\left(1-\left(1+\frac{1}{k_{c}}\right) X\right)$

com isso, é feito um balanço de energia no estado estacionário em termos do reagente A, para que se possa obter uma equação que descreva a variação de temperatura em função da conversão (Fogler, 2012; Stephanopoulos, 1983):

$\dot{Q}-\dot{W}-F_{A 0} \sum\left(\theta_{i} \widetilde{C_{p l}}\left(T-T_{0}\right)\right)-F_{A 0} X\left[\Delta H\left(T_{R}\right)+\Delta \widehat{C_{p}}\left(T-T_{R}\right)\right]=0$

Afirmando que a reação é conduzida adiabaticamente $(\mathrm{Q}=0)$, não existe trabalho $(\mathrm{W}=0)$ e a variação dos calores específicos é igual à zero, pois $\mathrm{C}_{\mathrm{pB}}=\mathrm{C}_{\mathrm{pA}}$, portanto, $\Delta \mathrm{C}_{\mathrm{p}}=0$. Rearranjando e substituindo essas condições na equação (8), tem-se:

$T=T_{0}+\frac{(-\Delta H) X}{\sum \theta_{i} \widetilde{C_{p l}}}$

sendo:

$\sum \theta_{i} \widetilde{C_{p l}}=\widetilde{C_{p A}}+\theta_{l} \widetilde{C_{p l}}=\left(141+\frac{0,1}{0,9} 161\right)=159,5 \frac{\mathrm{J}}{\mathrm{mol}} \cdot K$ 
e substituindo o somatório dos calores específicos (12) e as condições adicionais na equação (11), obtêm-se uma equação de temperatura em função da conversão (Fogler, 2012; Stephanopoulos, 1983):

$T=330+43,3 X$

Sabe-se que a constante cinética e de equilíbrio são dependentes da temperatura, portanto, é necessário utilizar as equações de Arrhenius (14) e Vant' Hoff (15) respectivamente, para avaliar como essas constantes variam com a temperatura.

$k=k\left(T_{1}\right) \exp ^{\left[\frac{E}{R}\left(\frac{1}{T_{1}}-\frac{1}{T}\right)\right]}$

$K_{c}=K_{c}\left(T_{2}\right) \exp ^{\left[\frac{\Delta H}{R}\left(\frac{1}{T_{2}}-\frac{1}{T}\right)\right]}$

substituindo as condições adicionais na equação (14) e (15), têm-se as equações das constantes cinéticas e de equilíbrio, respectivamente (Fogler, 2012; Stephanopoulos, 1983):

$k=31,1 \exp ^{\left[7906\left(\frac{1}{260}-\frac{1}{T}\right)\right]}$
$K_{c}=3,03 \exp ^{\left[-830,3\left(\frac{1}{333}-\frac{1}{T}\right)\right]}$

A concentração no tempo $t=0$ é $C_{A 0}=9,3 \mathrm{kmol} / \mathrm{m}^{3}$ e $C_{B 0}$ é igual à zero. É necessária a utilização do balanço de energia e da equação de Arrhenius nesse caso, devido não ter sido fornecido o valor da constante cinética $(\mathrm{k})$. Para realizar as simulações do reator PFR, será adotado o estado estacionário (Fogler, 2012; Stephanopoulos, 1983).

\section{Metodologia Proposta}

O sistema de análise da integridade estrutural proposto neste trabalho consiste em três módulos principais: aquisição de dados, censoriamento e monitoramento do sistema imunológico artificial.

O módulo de aquisição de dados é constituído pelo modelo numérico simulado pelo integrador numérico Runge Kutta de quarta ordem responsável por montar o banco de sinais do reator. Os sinais representam a resposta no tempo obtida pelo no módulo de entrada. O conjunto de sinais obtidos é usado como entradas para o sistema imunológico artificial.

O módulo de censoriamento foi executado o aprendizado ou treinamento inicial do sistema imune onde foram definidos os detectores próprios.

O conjunto de detectores próprios foi constituídos de 13 sinais que possuem a característica normal do reator rodando normalmente e 93 sinais que possuem as características com danos.

Assim, foi gerado o conjunto de detectores próprios aleatoriamente entre sinais normais e sinais com falhas sem repetir o mesmo sinal. Os dados foram comparados, ponto a ponto, com os detectores próprios. Quando um casamento é encontrado, o vetor aleatório é rejeitado, pois foi determinado características próprias. Caso contrário, é aceito, sendo armazenado no banco de dados como detector próprio. Este processo é ilustrado na Figura 4.

Depois de obtido o conjunto de detectores, o mesmo foi utilizado nas etapas do sistema de diagnóstico, tais como: o módulo de monitoramento do ASN. Depois de realizar o processo em modo on-line foi executado o processo de monitoramento em modo on-line. 


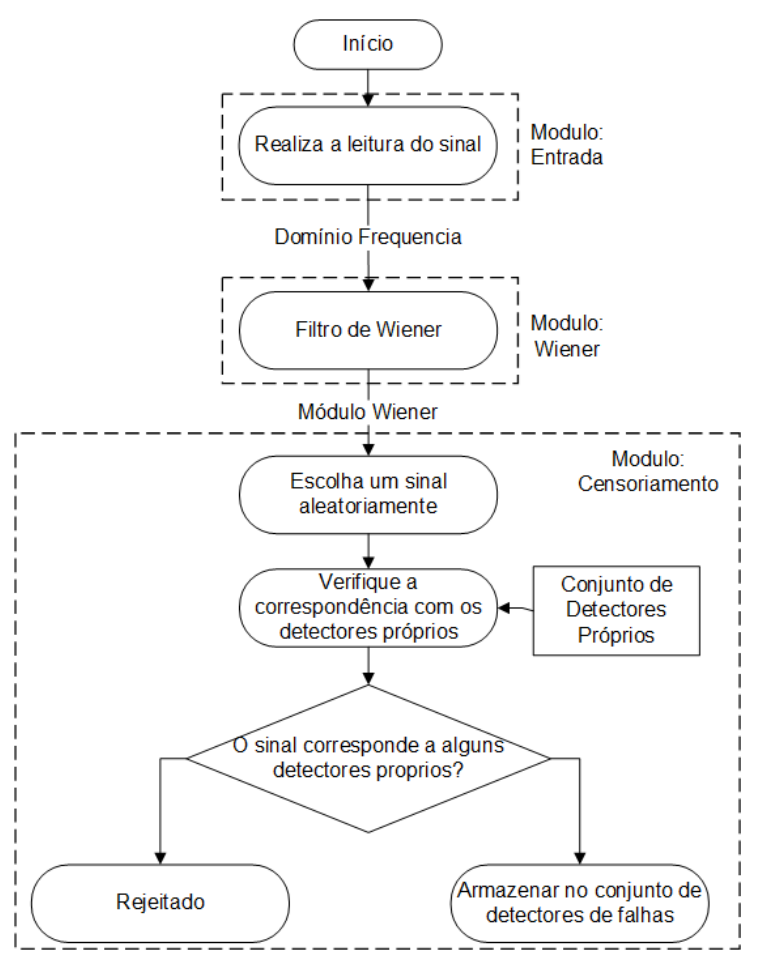

Figura 4. Metodologia proposta: Módulo de Censoriamento

O módulo de monitoramento é composto pelos módulos de entrada e de leitura dos sinais (via sistema de aquisição de dados), módulo do Filtro de Wiener, módulo de detecção de sinais que realizará a discriminação próprio/não-próprio para a identificação de danos, e o módulo de classificação, que irá caracterizar os danos estruturais. Todo esse processo de realizado é ilustrado na Figura 5.

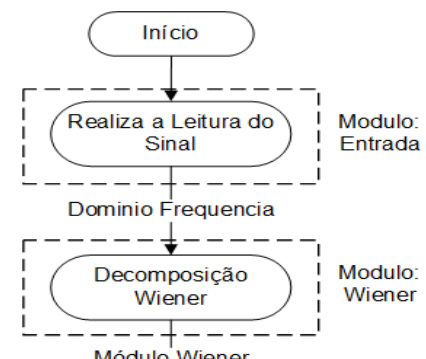

Módulo Wiener

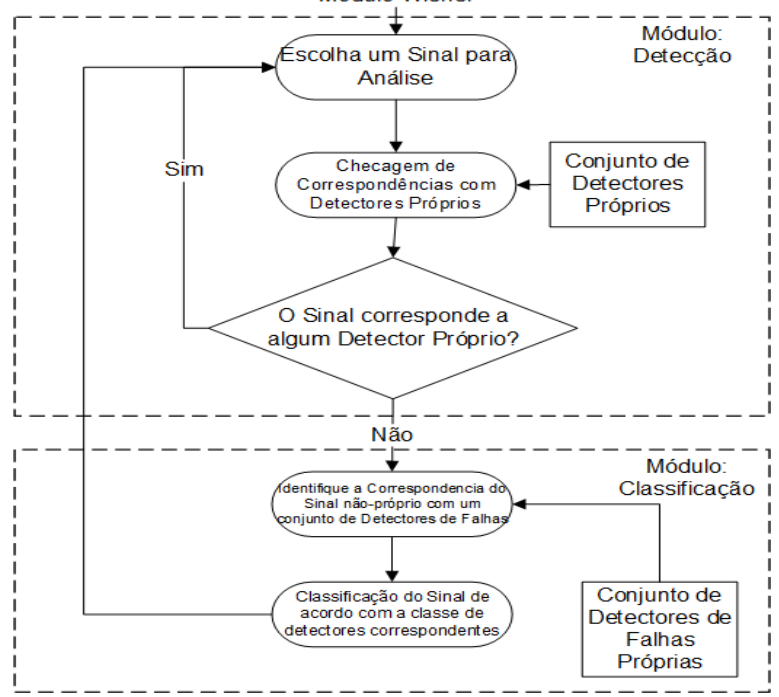

Figura 5. Metodologia proposta: Módulo de Monitoramento 


\section{Resultados}

Após a modelagem do reator conforme descrito na seção três, foi realizado as simulações das condições normais (baseline) e anormais (falha estrutural) no reator PFR em estado estacionário (volume inicial de 2.97 a 3,09), para o desbalanceamento do sistema, considerado falhas, sendo a variação do volume inicial de 2,03 a 2,9 e 4,01 a 4,05. Nestes casos foram gerados os sinais de cada fase e tratados separadamente. $\mathrm{O}$ resultado da coleta de dados simulados foram duas matrizes de $13 \times 16$ sinais sem falhas e 93x16 sinais com falhas. Um exemplo desse comportamento é ilustrado na Figura 6.

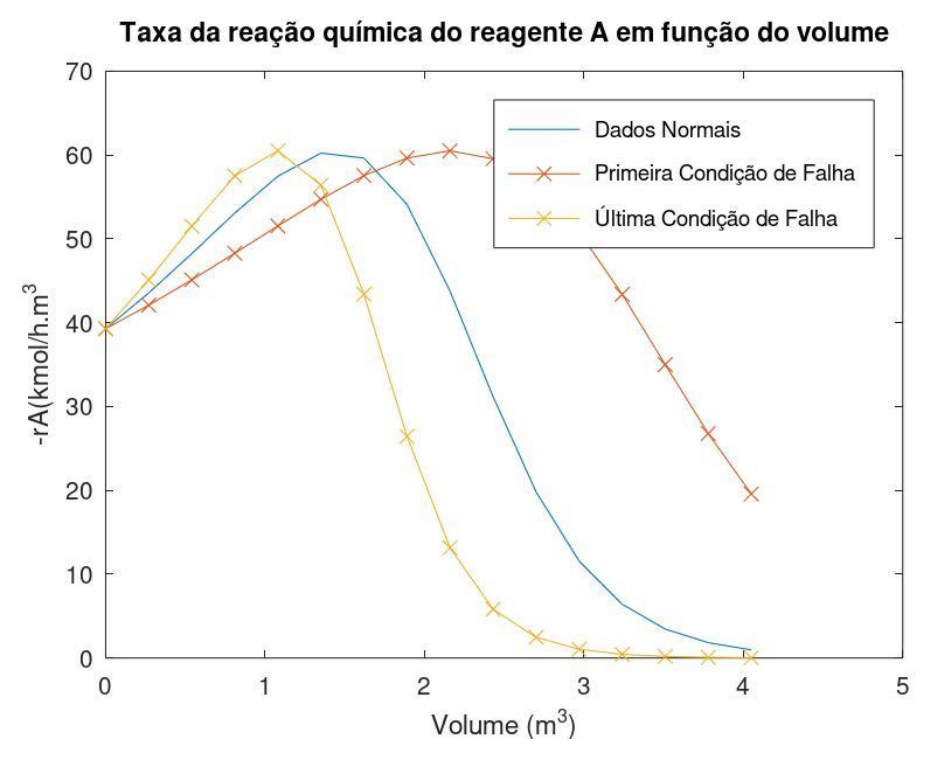

Figura 6. Banco de Dados: Condições normal e falhas do reator.

Após o tratamento dos sinais, o algoritmo atua analisando os sinais nas fases de censoriamento conforme ilustrado na figura 4 e monitoramento ilustrado na figura 5 , e utilizando o critério de casamento e afinidade para cada matriz de sinal sem falhas e a outra com falhas, respectivamente. $\mathrm{O}$ resultado da aplicação do algoritmo dos sistemas imunológicos artificiais utilizando reconhecimento de padrões analisou 106 sinais com 16 amostras e detectou que 13 dos sinais não contêm falhas, ou seja, identificação e reconhecimento assertivo de $100 \%$ das amostras de sinais sem falhas. Considerando que o algoritmo desenvolvido conseguiu classificar os sinais como sem falhas e com falhas, os 93 sinais com falhas foram criterizados conforme as condições do grau de severidade da falha e quantificado a probabilidade de ocorrência de falhas de cada sinal. A figura 7 mostra as divisões dos critérios da severidade e probabilidade da falha ocorrida no reator. 
Nivel Severidade Muito Alto

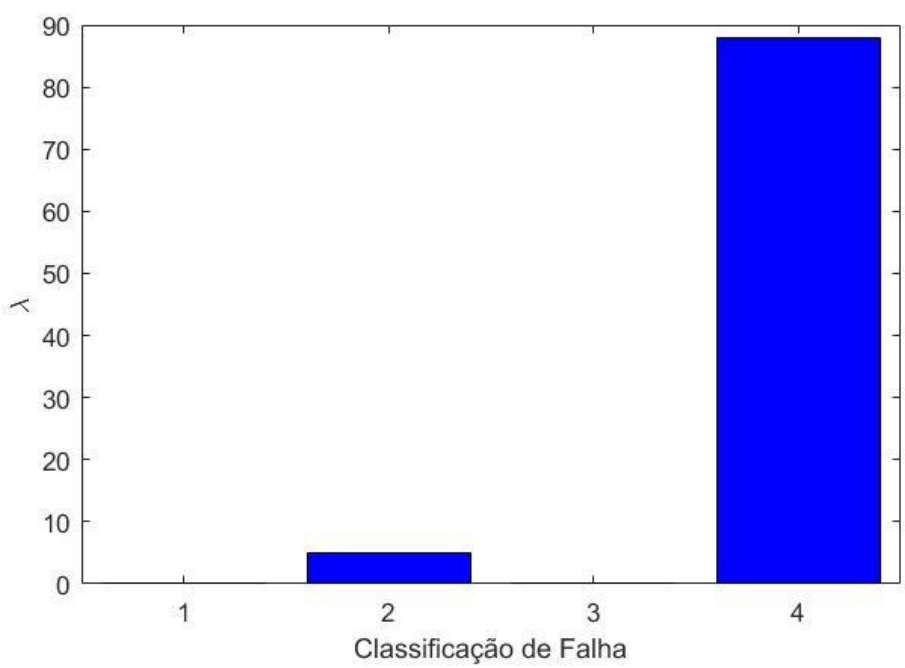

Figura 7. Critério do Sinal.

Note que a figura 7 ilustra o resultado da aplicação da variação do volume inicial que ocorre no desbalanceamento do reator, resultando na falha do sistema. No eixo horizontal, a classificação da probabilidade de falhas, o critério 1,2, 3 e 4, correspondem respectivamente a probabilidade de falha baixa, moderada, alta e muito alta. Já o eixo vertical, a quantidade de falhas $\lambda$, varia conforme a quantidade de sinais encontrados pelo algoritmo. Nota-se ainda que, existe apenas um nível de severidade de falha, sendo este respectivamente, o muito alto.

Portanto, de uma forma geral, o reator ao variar o volume inicial teve uma concentração maior de sinais no nível de severidade muito alto. Já a variação da quantidade de sinais distribuídos na probabilidade de falhas muito alta, dos grupos de nível de severidade médio e muito alto, demonstra que o reator pode tender a um comportamento de quebra, dado a continuidade das variações de volume e intensidade de desbalanceamento. Isso comprova que o desequilíbrio do sistema pode ser detectado pelo sistema de monitoramento.

\section{Conclusões}

O prognóstico por sensoriamento de reatores é importante em um sistema de gerenciamento da manutenção, considerando ainda os conceitos da indústria 4.0, pois mostram a realidade do comportamento do componente, possibilitando uma tomada rápida de decisão. Além disso, o componente quando monitorado, permite antecipar paradas em linha, baseado na condição da vida útil, melhorando a segurança e contribuindo com a prevenção da manutenção em técnicas como a total productive maintenance TPM e SHM.

Neste artigo foi proposta uma nova abordagem baseados no conceito da indústria 4.0 para realizar a análise de integridade estrutural de um reator, em que se empregou o sistema imunológico artificial apresentando excelentes resultados, obtendo um índice de acerto de 100\% para a melhor configuração do sistema. Esta proposta é uma composição do sistema imunológico artificial com o reator, que tem por objetivo melhorar a capacidade de diagnóstico do sistema inteligente. Assim, a partir da aquisição e processamento dos sinais, aplica-se o sistema imunológico artificial para identificar e caracterizar as falhas.

Considerando que o objetivo deste trabalho foi o de desenvolver uma nova metodologia de prognose e detecção de falhas utilizando sistemas inteligentes aplicados a um reator químico, pode-se concluir que o sistema imunológico artificial proposto é eficiente, confiável e robusto na análise de integridade de estruturas. 


\section{Agradecimentos}

O segundo autor agradece ao Conselho Nacional de Desenvolvimento Científico e Tecnológico (Proc. N ${ }^{o} 312972$ / 2019-9) e a Fundação de Amparo à Pesquisa do Estado de São Paulo (FAPESP Proc. 2019 / 10515-4) pelo apoio ao projeto e os autores agradecem ao Laboratório de Sistemas Complexos (SISPLEXOS) onde o projeto foi desenvolvido.

\section{Referencias}

Bradley, D.W., Tyrrell, A.M., (2002). Immunotronics-novel finite-state-machine architectures with built-in self-test using self-nonself differentiation. IEEE Transactions on Evolutionary Computation, New York, v. 6, p. 227-238.

Chen, Y.; Li, Y. (2018). Computational Intelligence Assisted Design: In Industrial Revolution 4.0, CRC Press, Boca Raton. 527p.

Farrar, C.R., Worden, K., (2013). Structural Health Monitoring: A Machine Learning Perspective, Chichester, John Wiley. 643p.

Fogler, H. S. (2012). Elementos De Engenharias Das Reações Químicas. 4. Ed. Rio de Janeiro: LTC. 888p.

Forrest, S., Perelson, A. S., Allen, L., Cherukuri, R. (1994). Self-nonself discrimination in a computer. Proceedings of 1994 IEEE Computer Society Symposium on Research in Security and Privacy, Oakland, v.1, n.1, pp. 202-212. doi:10.1109/RISP.1994.296580.

Franco, V. R.; Bueno, D. D.; Brennan, M. J.; Cavalini Jr., A. A.; Gonsalez, C. G.; Lopes JR., V. (2009). Experimental damage location in smart structures using Lamb waves approaches. In: Brazilian Conference on Dynamics, Control and Their Applications - DINCON. p. 1-4.

Kelly, R. (2019). Constructing Leadership 4.0 Swarm Leadership and the Fourth Industrial Revolution, Springer Nature, Switzerland. 227p.

Lima, F. P. A., Chavarette, F. R., Souza, S. S. F., Lopes, M. L. M., (2017). Monitoring and Fault Identification in Aeronautical Structures Using an Wavelet-Artificial Immune System Algorithm, In: Tephen Ekwaro-Osire, Aparecido C. Gonçalves, Fisseha M. Alemayehu. (Org.). Probabilistic Prognostics and Health Management of Energy Systems. 12ed. São Paulo: Springer International Publishing, v. 1, p. 1-17. doi: http://dx.doi.org/10.1007/978-3319-55852-3_13.

Moro, T.C.; Chavarette, F.R.; Roefero, L.G.P.; Outa, R. (2019). Detecção de falhas estruturais de um prédio de dois andares utilizando Sistema Imunológico Artificial. Colloquium Exactarum, v. 11, p. 73-84. doi: https://doi.org/10.12732/ijpam.v97i1.9.

Outa, R., Chavarette, F.R.; Toro, P.; Goncalves, A.C.; Dos Santos, L.H., (2021). Prognosis and Detection of Experimental Failures in Open Field Diesel Engines Applying Wiener's Artificial Immunological Systems, Journal of Applied and Computational Mechanics, v.7, n.1. doi: 10.22055/jacm.2020.34972.2525.

Outa, R.; Chavarette, F.R.; Mishra, V.N.; Goncalves, A.C.; Roefero, L.G.P.; Moro, T.C. (2020). Prognosis and fail detection in a dynamic rotor using artificial immunological system. Engineering Computations, v. 37, n.9, p. 3127-3145. doi: doi.org/10.1108/EC-08-20190351.

Popkova, E.G.; Ragulina, Y.V.; Bogoviz, A.V. (2019). Industry 4.0: Industrial Revolution of the $21^{\text {st }}$ Century: Studies in Systems, Decision and Control, Springer International Publishing, Gewerbestrasse, 249p.

Stephanopoulos, G. (1983). Chemical Process Control: An Introduction to Theory and Practice, Prentice Hall, New Jersey. 704p. 